\title{
Characterization of cracking in pavement distress using image processing techniques and k-Nearest neighbour
}

\author{
A. Ibrahim ${ }^{1}$, M.K.Osman ${ }^{2}$, N.A.M. Yusof ${ }^{3}$, K. A. Ahmad ${ }^{4}$, N.H. Harun ${ }^{5}$, R.A.A Raof ${ }^{6}$ \\ ${ }^{1}$ Faculty of Civil Engineering, Universiti Teknologi MARA, Malaysia \\ ${ }^{2,4}$ Faculty of Electrical Engineering, Universiti Teknologi MARA, Malaysia \\ ${ }^{3}$ Electrical Engineering Department, Politeknik Tuanku Sultanah Bahiyah, Malaysia \\ ${ }^{5}$ Data Science Research Lab, School of Computing, Universiti Utara Malaysia, Malaysia \\ ${ }^{6}$ School of Computer \& Communication Engineering, Universiti Malaysia Perlis, Malaysia
}

\begin{tabular}{l} 
Article Info \\
\hline Article history: \\
Received Sep 28, 2018 \\
Revised Nov 25, 2018 \\
Accepted Dec 7, 2018 \\
\hline Keywords: \\
Fuzzy k-nearest neighbour \\
Image processing \\
k-nearest neighbour \\
Pavement distress analysis
\end{tabular}

Article Info

Article history:

Received Sep 28, 2018

Revised Nov 25, 2018

Accepted Dec 7, 2018

Pavement distress analysis

\begin{abstract}
This study presents characterization of cracking in pavement distress using image processing techniques and k-nearest neighbour $(\mathrm{kNN})$ classifier. The proposed semi-automated detection system for characterization on pavement distress anticipated to minimize the human supervision from traditional surveys and reduces cost of maintenance of pavement distress. The system consists of 4 stages which are image acquisition, image processing, feature extraction and classification. Firstly, a tool for image acquisition, consisting of digital camera, camera holder and tripod, is developed to capture images of pavement distress. Secondly, image processing techniques such as image thresholding, median filter, image erosion and image filling are applied. Thirdly, two features that represent the length of pavement cracking in $\mathrm{x}$ and $\mathrm{y}$ coordinate system namely delta_x and delta_y are computed. Finally, the computed features is fed to a kNN classifier to build its committee and further used to classify the pavement cracking into two types; transverse and longitudinal cracking. The performance of $\mathrm{kNN}$ classifier in classifying the type of pavement cracking is also compared with a modified version of kNN called fuzzy kNN classifier. Based on the results from images analysis, the semi-automated image processing system is able to consistently characterize the crack pattern with accuracy up to $90 \%$. The comparison of analysed data with field data shows good agreement in the pavement distress characterization. Thus the encouraging results of semi-automated image analysis system will be useful for developing a more efficient road maintenance process.
\end{abstract}

Copyright $(2019$ Institute of Advanced Engineering and Science. All rights reserved.

\section{Corresponding Author:}

Anas Ibrahim,

Faculty of Civil Engineering,

Universiti Teknologi MARA (UiTM) Kampus Pulau Pinang,

13500 Permatang Pauh, Pulau Pinang, Malaysia.

Email: ceanas@ppinang.uitm.edu.my

\section{INTRODUCTION}

Pavement distress is a condition of pavement that shown imperfection on the surface of pavement. Cracking is one of the most common and important types of asphalt pavement distress [1-2]. Generally, cracking distress can be divided into three main types which are longitudinal, alligator and transverse cracking [3-5]. Pavement maintenance is generally identified as work accomplished to preserve or extend the pavement's service life until major rehabilitation or complete reconstruction is performed. It is classified by function as either routine or preventive. In Malaysia, the pavement distress analysis is normally conducted manually using visual inspection by road maintenance operator. The manual system is 
costly, hazardous to the operator and expensive due to the longer hours of assessment by operator [6]. Therefore there is a need to develop a more efficient automated system to perform pavement distress analysis. Hence the application of artificial intelligence (AI) was explored to assist in characterization of pavement distress. AI system needs very minimal labour to verify the cracking pavement distress that allows faster decision to be made in selecting suitable treatment method.

From the previous works, most of existing methods of AI for identifying defects on pavement surface are based on characteristics of crack. They consider that crack pixels are darker than the ones that surround them. Different researcher used fixed threshold to detect dark pixels (cracks). As a key approach of crack detection, thresholding can affect the efficiency of image segmentation during the image processing [7]. Although there are many methods to compensate the crack and remove the noise, a good thresholding method is needed to obtain a clear binary image. A several image enhancement techniques, smoothing, low-pass filtering, sharpening were compared by research of Rababaah [7 and the best result was using the Median filter after thresholding. Median filtering can be used to reduce the noise while preserving much of the details in the image. Essentially, there are a few ways to controlled condition of an image at image acquisition stage. For example, [7] applied the technique on pavement survey vehicles by modified the back of the Mobile Laboratory with attached a digital camera, as depicted in Figure 1. It permits high speed surveys of geo-referenced digital images of the pavement to be made by integrating the GPS and the digital image data acquisition system.

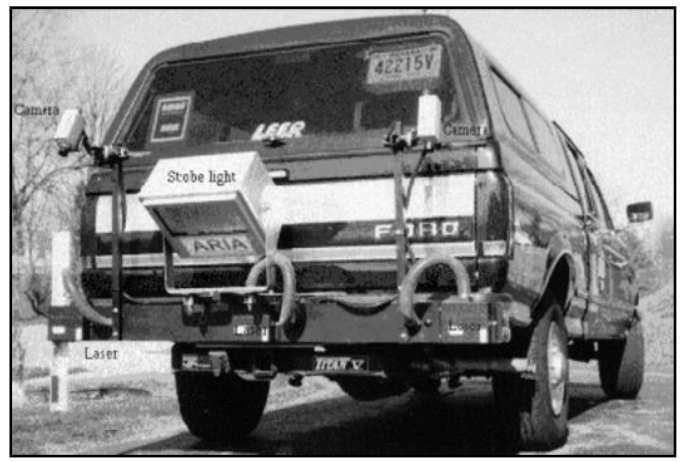

Figure 1. Pavement survey vehicle [6]

Another method to extract binary image is to use an adaptive fuzzy thresholding where it can identify pavement distress from original images. Saar and Talvik [8] found the main idea behind this method is based on the fact that crack pixels in pavement images are both continuous and darker than their surroundings. Figure 2 shows an example of threshold process where at the left is an original image and at the right is image after threshold process.

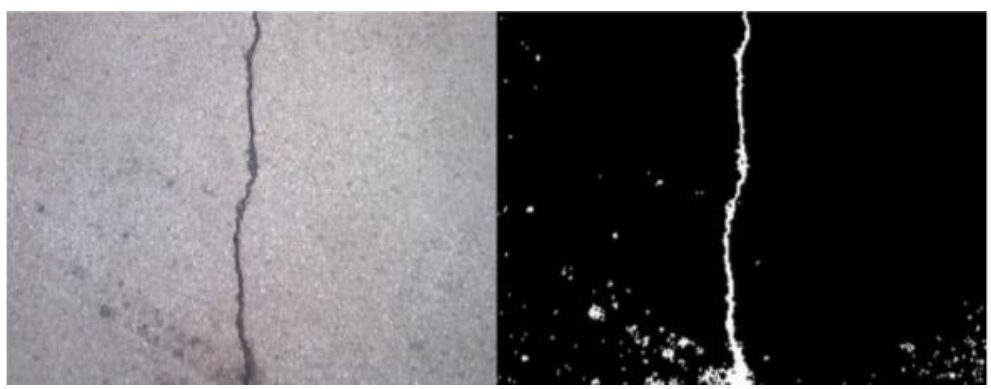

Figure 2. Original (left) and threshold (right) image [7]

Most recent, Banharnsakun [6] combines artificial bee colony (ABC) algorithm and artificial neural network (ANN) called "ABC-ANN" for crack type classification. Thresholding method based on ABC algorithm was initially used to partition captured images into two regions, distressed and non-distressed regions based on a thresholding method. Then, three features namely vertical distress measure, horizontal 
distress measure and total number of distress pixels, are computed from the distressed region. Finally, an ANN is used to classify these input features into three types, transversal crack, longitudinal crack, and pothole. By using the proposed method, the author claimed to achieve $20 \%$ increase compared to the existing algorithm.

This study proposed to use k-nearest neighbor $(\mathrm{kNN})$ and fuzzy k-nearest neighbor (fuzzy $\mathrm{kNN}$ ) to classify crack types. Both classifiers were selected due to very simple, require less data and reported to have highly efficient and effective in solving classification problems [9-11].

\section{RESEARCH METHOD}

The research methodology consists of four (4) parts namely image acquisition, image processing, feature extraction and classification. Figure 3 shows the flowchart methodology of this study.

\subsection{Image Acquisition}

In this process, pictures were taken by using a digital camera during daylight with its optical perpendicular to the road surface and its lateral edge parallel to the axis. Image of pavement distress was collected from surrounding area of UiTM Cawangan Pulau Pinang and arbitrary streets in Pulau Pinang which will be used for this project. To get a good result, the image must be clear image with cracks without any obstacle such as shadow, sand inside the crack or any unwanted things that will disturb the image processing. Referring to [7], to keep the picture maintain consistent, reliable and systematic, some precaution must be considered during image acquisition:

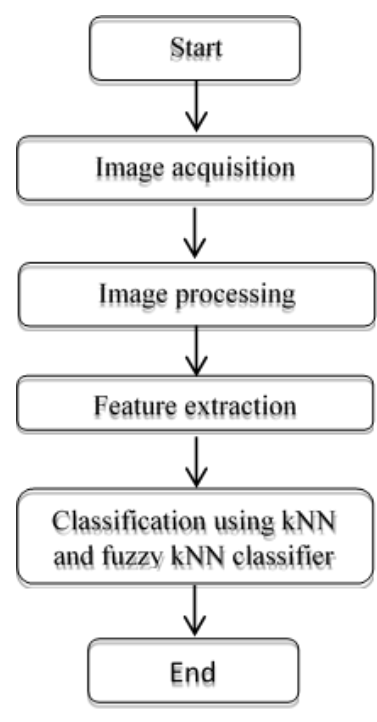

Figure 3. Flowchart of the proposed research methodology

a) Illumination: For better quality, image capturing is conducted during a bright day and at similar timing for consistency. Image brightness and contrast will affect the process reading to detect type of crack. Therefore, illumination is an important to ensure the image will captured with a good quality for image processing data.

b) Distance from the camera to the pavement: The distance is fixed at the same level during taking a picture of pavement distress. It will affect the size of crack and the relative gabs between crack object to detect and classify the type of crack.

c) Expert Validation: Get a consulted and approved from the expert to develop a correct way of data collection technique to get an accurate result.

Based on the abovementioned precautions, the tool has been built as shown in Figure 4. In order to obtain consistent, reliable and efficient images collection, the digital camera is mounted on a camera holder with consistent height distance of $80 \mathrm{~cm}$ from the pavement surface. The image capturing process was performed during daylight at similar timing from 8.00 am to $11.00 \mathrm{am}$. For a better result, data collection at noon starting from $12.00 \mathrm{pm}$ until $3.00 \mathrm{pm}$ was avoided to minimized shadow interruption. 


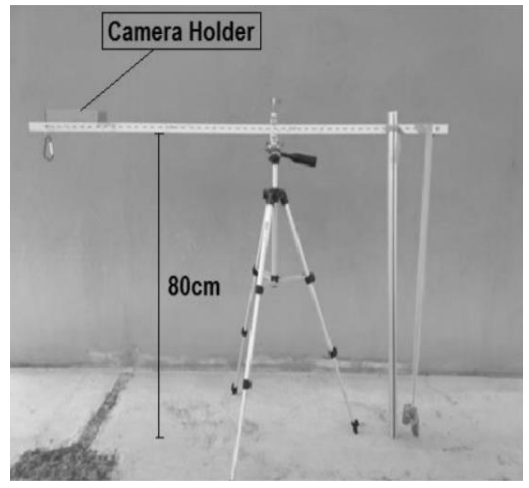

Figure 4. Tool for image acquisition

\subsection{Image Processing}

Image processing is an important stage for this application system with several processes that must be through to get a result. A simple image processing was unable to give an accurate reading to the image that we want to process due to the size of image is too large, non-uniform lighting or weather conditions or the contrast of image is in low quality. Just because of this problem crack that presence on the road surface cannot be detect or classify. Hence, image processing will be used to enhance the image so that it can be process based on what we want for the result. The image processing techniques was implemented using MATLAB Image Processing Toolbox.

The proposed image processing steps in this study is described as a process that involved RGB to grayscale image conversion, image segmentation, median filter, image erosion, image filled and image remove small area. The analysis of image was start with uploading images to MATLAB program. Next, the RGB to grayscale image conversion was applied to reduce computational quantity and complexity during thresholding.

Image segmentation means process to divide the image into object and background. In this study manual image thresholding was selected due to the problems of images with different condition and illuminations. The process is achieved by gradually increase the threshold value, $t$ from zero to the value that can visually eliminate most of the background and retaining most of the object (pavement crack) in the image. Then, the median filter takes place which means process of reduces of noise of image. Subsequent, image erosion was implemented to the resultant image and image filled was used to fill a holes on object of image to get a sharp of object image. Lastly, the process of remove unwanted image using image remove small area and that is the resultant image for image processing stage.

\subsection{Feature Extraction}

This process is considered as process of converting the given data into classifier acceptable format and for the classification task are computed for the normalized images. The image is to be represented into relevant features for recognition process, so that the classifier is applied to recognize the object. This feature is useful in finding the length of major axis and minor axis by propose delta_ $x$ and delta_ $y$ to classify type of crack. If the size of delta_ $x$ is greater than delta_y it is means the type of crack pavement is transverse crack. Other than that, if the size of delta_y is greater than delta_ $x$ it is means the type of crack pavement is longitudinal crack. Figure 5 shows the MATLAB source code to calculate delta_ $x$ and delta_y. The program starts by finding all the pixels coordinates that representing the object (the white pixel) in BW image. Then, the maximum and minimum coordinate of $\mathrm{y}$-axis and $\mathrm{x}$-axis is determined using $\max ()$ and $\min ()$ functions. Finally the delta_x and delta_y are determined by finding the difference between the maximum and minimum coordinates.

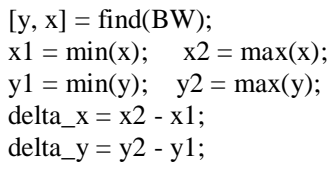

Figure 5. MATLAB source code to determine delta_x and delta_y 


\subsection{Crack Classification using kNN and Fuzzy kNN Classifier}

The last part is classification stage which is performed using $\mathrm{kNN}$ and fuzzy $\mathrm{kNN}$ classifiers. The kNN classifier is one of the classifier that are very simple to understand but work incredibly well in practice and become well known nowadays in the pattern of classification procedure. This method has been used in many applications in the field of data removal, numerical pattern recognition and many others. The kNN classifier is widely use in the pattern recognition community due to its good performance in practical application. KNN classifies an object based on closest training examples by a majority vote of its neighbour.

The fuzzy kNN classifier is a modified version of kNN classifier. The fuzzy k-nearest neighbour algorithm assigns class membership to a sample vector rather than assigning the vector to a particular class as in the $\mathrm{kNN}$ [13]. No arbitrary assignment is the advantages are made by the algorithm. In addition, a level of assurance to accompany the resultant classification had been provided from the vector's membership values. In both methods, the number of nearest neighbor, $k$ is usually set to odd values $(3,5,7,9$, etc. $)$ to ensure there is no equal number of example is selected.

In this study, the kNN and fuzzy kNN target (cracking type) is assigned 1 as longitudinal crack and 2 as transverse crack. Example of longitudinal and transverse crack is illustrated in Figure 6. Both types are the most common pavement distress type encountered during road survey. Meanwhile, the number of nearest neighbor $k$ is set similar to 3 for both $\mathrm{kNN}$ and fuzzy $\mathrm{kNN}$ classifiers. The value was determined experimentally and found to be the sufficient in classifying the pavement cracking types.

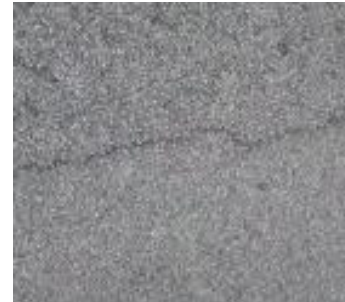

(a) Transverse crack

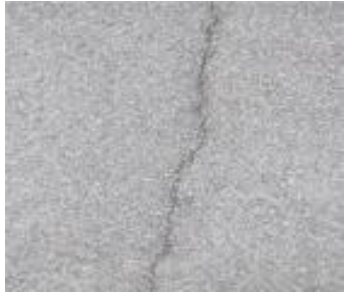

(b) Longitunidal crack

Figure 6. Types of crack classified using kNN and fuzzy kNN algorithm

\section{RESULTS AND ANALYSIS}

During image acquisition step, a total of 150 images (75 transverse crack and 75 longitunidal crack) were captured in different areas. Example of the captured images are shown in Figure 7.
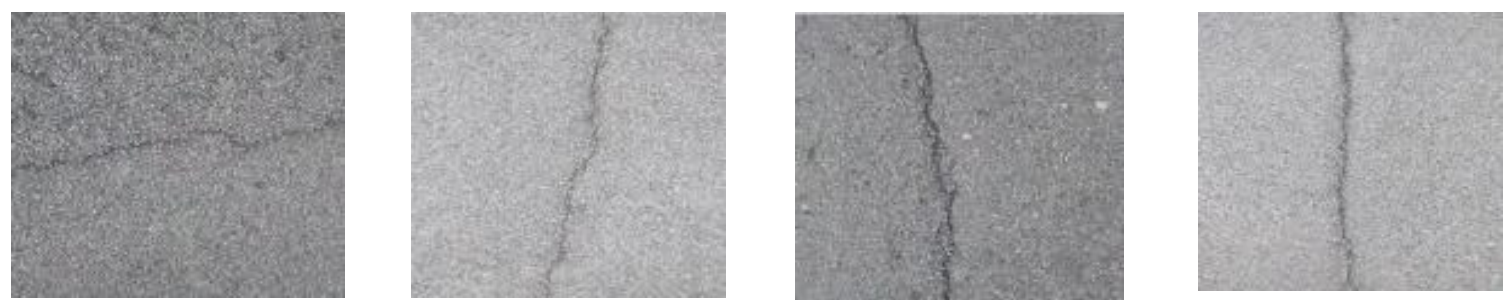

Figure 7. Examples pavement crack images capture using the image acquisition system.

It can clearly be seen that the pavement crack pixels are usually darker than the surrounding pixels. However, the surrounding pixels were found to have different intensities in different images. This is due to inconsistency in lighting conditions and non-uniform pavement surface. The problems will result in difficulty in achieving satisfactory image segmentation results and further lead to misclassification of pavement cracks.

The study proposed to use manual thresholding to overcome the problem. The manual thresholding is performed by gradually increase the threshold value, $t$ from zero to the value that can visually eliminate most of the background and retaining most of the object (pavement crack) in the image. Figure 8(a) gives an example of pavement crack image and Figure $8(\mathrm{~b})$ is the resultant image after applying the manual threshold $(t=0.29)$. Table 1 gives the minimum, maximum and average threshold value selected during the manual 
thresholding process. These value were obtained thorough analysis of 150 images, as mentioned previously. Table 1. The Minimum, Maximum and Average Threshold Value for 150 Crack Images

\begin{tabular}{cccc}
\hline Type of crack & Minimum & $\begin{array}{c}\text { Threshold value, } t \\
\text { Maximum }\end{array}$ & Average \\
\hline Longitunidal & 0.25 & 0.39 & 0.35 \\
Tranverse & 0.26 & 0.38 & 0.34 \\
& & Average & 0.35 \\
\hline
\end{tabular}

It can be seen that the manual image thresholding has partially removed the background pixels with higher intensity values. Unfortunately, the method was unable to eliminate the background pixels having intensity similar or lower than the pavement crack intensities. Therefore, a median filter was applied to reduce the image noise. Subsequent, image erosion was implemented to the resultant image and image filled was used to fill a holes on object of image to get a sharp of object image. Lastly, the process of remove unwanted image using image remove small area and that is the resultant image for image processing stage. Figure 8 give an example of a crack image and the results after applying the image processing techniques.

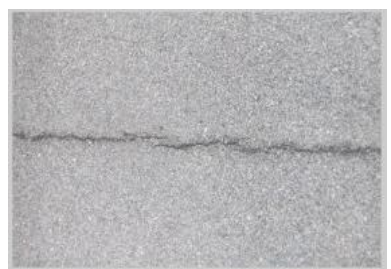

(a) Original image

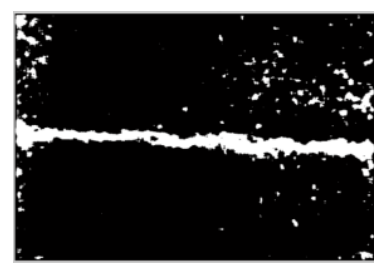

(c) Median filter

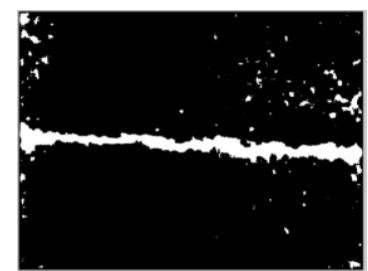

(e) Filled image

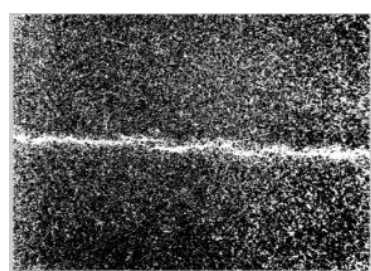

(b) RGB to grayscale image conversion and manual threshold $(\mathrm{t}=0.29)$

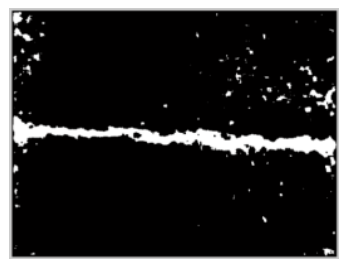

(d) Image erosion

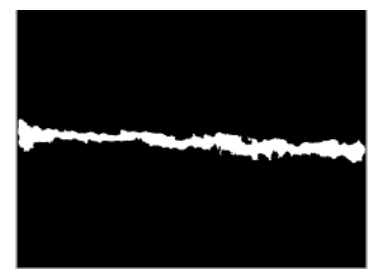

(f) Remove small area image

Figure 8. Example of pavement cracking image and its resultant images after applying image processing techniques

Feature extraction was applied to the processed images to extract pavement crack information. Two features called the length of major axis and minor axis by propose delta_ $x$ and delta_ $y$ were extracted. Both features were then fed into kNN classifier to identify the type of crack. Table 2 gives examples of crack, value of delta_x and delta_y and type of crack classified by the kNN classifier.

For each crack type, 25 randomly selected images was used to evaluate the classification performance of $\mathrm{kNN}$ classifier while the remaining images were used to build the $\mathrm{kNN}$ committee. In this study, the number of nearest neighbor, $k$ is set to 3 for all analyses. Classification performance of kNN classifier was evaluated in term of accuracy and processing time, and further compared with the fuzzy kNN classifier. Processing time refers to the time interval between the image features extraction and classification step. The image processing step was not accounted for processing time due its manual processing. Table 3 summarises the achievement of the classifiers. For a fair comparison, the number of nearest neighbor $k$ for fuzzy $\mathrm{kNN}$ classifier is set to 3 , similar to the $\mathrm{kNN}$ classifier. 
Referring to Table 3, classification accuracy of kNN classifier was found similar to the fuzzy kNN classifier. Three (3) logitunidal and two (2) tranverse crack images was wrongly classified by both classifier. Further investigation on the resultant image found that the presence of noise after the image processing step has led to misclassification of the crack type. However, classification using kNN classifier is more efficient since its processing time is slightly lower than the fuzzy kNN classifier, and thus chosen as the final classifier.

Table 2. Examples of Pavement Crack, Value of delta_x and delta_y and Type of Crack Classified by kNN Classifier

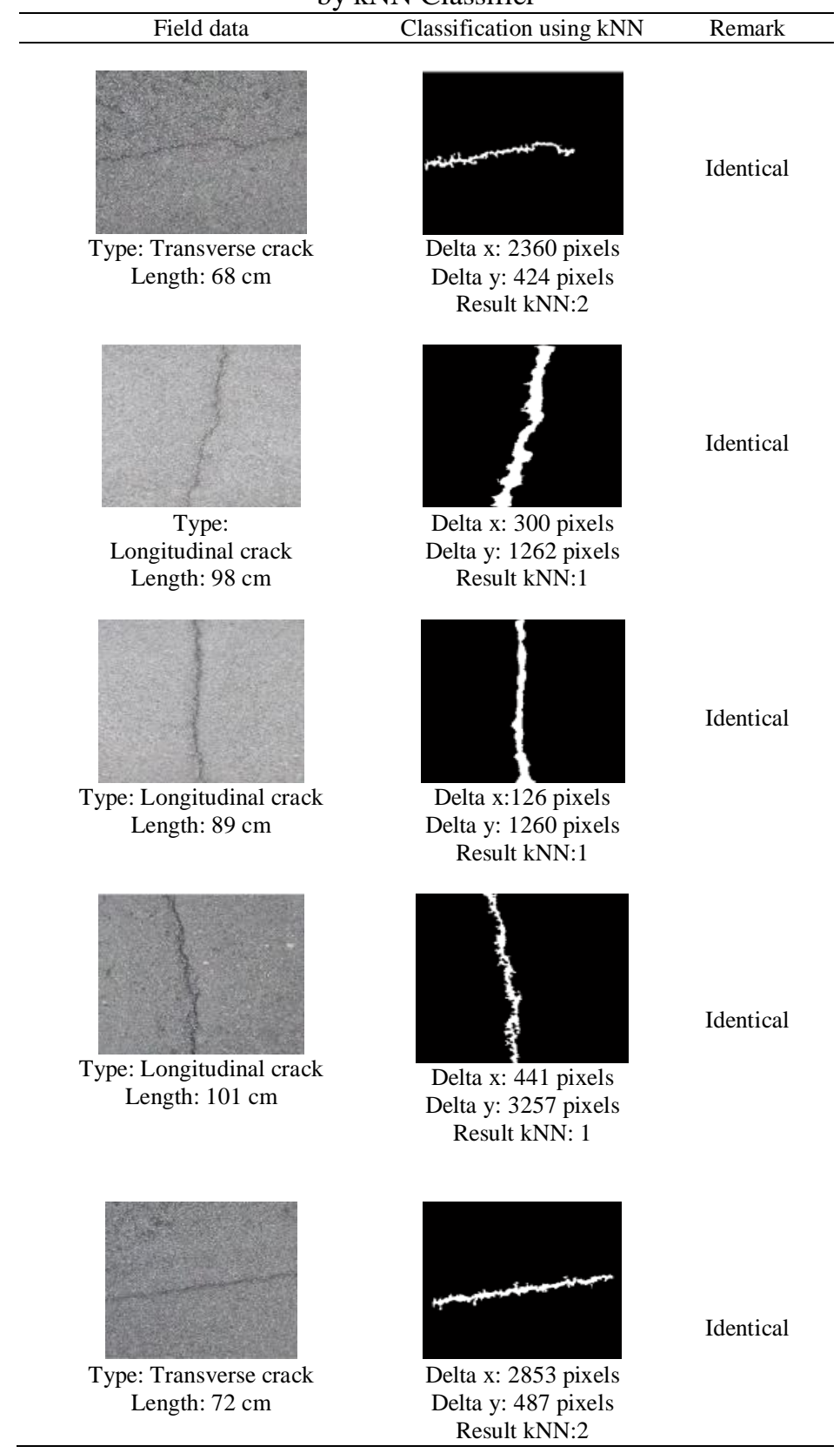

Table 3. Classification Performance using kNN and Fuzzy kNN Classifier for Longitudal and Tranverse Crack

\begin{tabular}{|c|c|c|c|c|}
\hline \multirow[b]{2}{*}{ Type of crack } & \multicolumn{2}{|c|}{ kNN } & \multicolumn{2}{|r|}{ FkNN } \\
\hline & $\begin{array}{c}\text { Classiffication accuracy } \\
(\%)\end{array}$ & $\begin{array}{l}\text { Average processing } \\
\text { time }(\mathrm{s})\end{array}$ & $\begin{array}{l}\text { Classiffication } \\
\text { accuracy }(\%)\end{array}$ & $\begin{array}{c}\text { Acerage processing time } \\
\text { (s) }\end{array}$ \\
\hline Longitunidal & 88.00 & 5.1464 & 88.00 & 7.2330 \\
\hline Tranverse & 92.00 & 6.0641 & 92.00 & 9.0598 \\
\hline
\end{tabular}




\section{CONCLUSION}

Average $\quad 90.00$

8.1464

A semi-automated pavement distress characterization analysis using image processing and AI was successfully developed based on the results obtained from images acquisition and analysis. The basic tool that was used in images capturing process has significantly improved the consistency and quality of photo taken at the site. The program development using MATLAB software was success and able to analyse cracking images with good consistency and reliability.

The evaluation of the effectiveness of Artificial Intelligence by detecting of cracking in pavement distress was completely achieved by using between kNN and fuzzy kNN classifier. The effectiveness of this research includes processing time to classify the pavement distress. On average, this study consumes not more than a minute of processing time to detect on cracking of pavement distress. Lower images processing time and the capability of repeating the same procedures on a thousand of images without fatigue could be the main key in replacing human limited capability in images analysing. The verification of the results obtained from AI analysis based on comparison with the field data produced an excellent agreement. Thus, the semi-automated AI system was able to identify the cracking pattern from pavement distress based on the images analysis using $\mathrm{kNN}$ and fuzzy kNN classifier.

\section{ACKNOWLEDGEMENTS}

The author would like to thank to Highway and Traffic Engineering Lab and Advanced Rehabilitation Engineering in Diagnostic and Monitoring Research Group (AREDiM), UiTM for providing facilities in the area of study. Also, appreciation goes to the Ministry of Higher Education (MOHE) for giving great privilege in providing scholarship as inspiring encouragement for the success of this research.

\section{REFERENCES}

[1] C. Koch, K. Georgieva, V. Kasireddy, B. Akinci, and P. Fieguth, "A review on computer vision based defect detection and condition assessment of concrete and asphalt civil infrastructure", Advanced Engineering Informatics, 29(2), 196-210, 2015.

[2] H. Zakeri, F.M. Nejad and A. Fahimifar. "Image Based Techniques for Crack Detection, Classification and Quantification in Asphalt Pavement: A Review." Archives of Computational Methods in Engineering 1-43, (2016).

[3] M. Gopikrishna, G. Baladi, T. Dawson, M. Prohaska, and K. Thomas. "Impacts of Maintenance Treatments on the Life Cycle Pavement Condition and Distress of the LTPP SPS-3 Test Sections." In Transportation Research Board 95th Annual Meeting, no. 16-5077. 2016. B. Smith, "An approach to graphs of linear forms, 2016.

[4] D. Wang, C.F. Augusto, M. Goeke, M.P. Wistuba, and Y. Tsai. "A multi-scale diagnosis model for asphalt pavement cracking in China." In Functional Pavement Design: Proceedings of the 4th Chinese-European Workshop on Functional Pavement Design (4th CEW 2016, Delft, The Netherlands, 29 June-1 July 2016, 2016.

[5] L. Li, and K.C.P. Wang. "Bounding Box-Based Technique for Pavement Crack Classification and Measurement Using $1 \mathrm{~mm}$ 3D Laser Data." Journal of Computing in Civil Engineering 30, no. 5, 2016.

[6] A. Banharnsakun. "Hybrid ABC-ANN for pavement surface distress detection and classification." International Journal of Machine Learning and Cybernetics 8, no. 2, 2017.

[7] H. Rababaah, "Asphalt pavement crack classification: A comparative study of three AI approaches: Multilayer perceptron, genetic algorithms and self-organizing maps." $\mathrm{PhD}$ diss., Indiana University South Bend, 2005.

[8] T. Saar and O. Talvik. "Automatic asphalt pavement crack detection and classification using neural networks." In Electronics Conference (BEC), 2010 12th Biennial Baltic, pp. 345-348. 2010.

[9] P. Lv, P. Yang, Y. Dong and L. Gu. "BALLKNN: An efficient and scalable KNN based on Euclidean similarity." 2016 IEEE International Joint Conference on Neural Networks (IJCNN), pp. 5141-5148., 2016.

[10] P. Thamilselvan J.G.R. Sathiaseelan. "A comparative study of data mining algorithms for image classification." International Journal of Education and Management Engineering 2, 2015.

[11] S.S. Tabrizi, and N. Cavus. "A hybrid KNN-SVM model for Iranian license plate recognition." Procedia Computer Science 102:588-594, 2016.

[12] A. Shubair, S. Ramadass and A.A. Altyeb. "kENFIS: kNN-based evolving neuro-fuzzy inference system for computer worms detection." Journal of Intelligent \& Fuzzy Systems 26, no. 4, 2014.

[13] S. Sajadi, and M. Amirfakhrian. "Fuzzy K-Nearest Neighbor Method to Classify Data in a Closed Area" International Journal of Mathematical Modelling \& Computations 3, no. 2, 109-114, 2013. 


\section{BIOGRAPHIES OF AUTHORS}

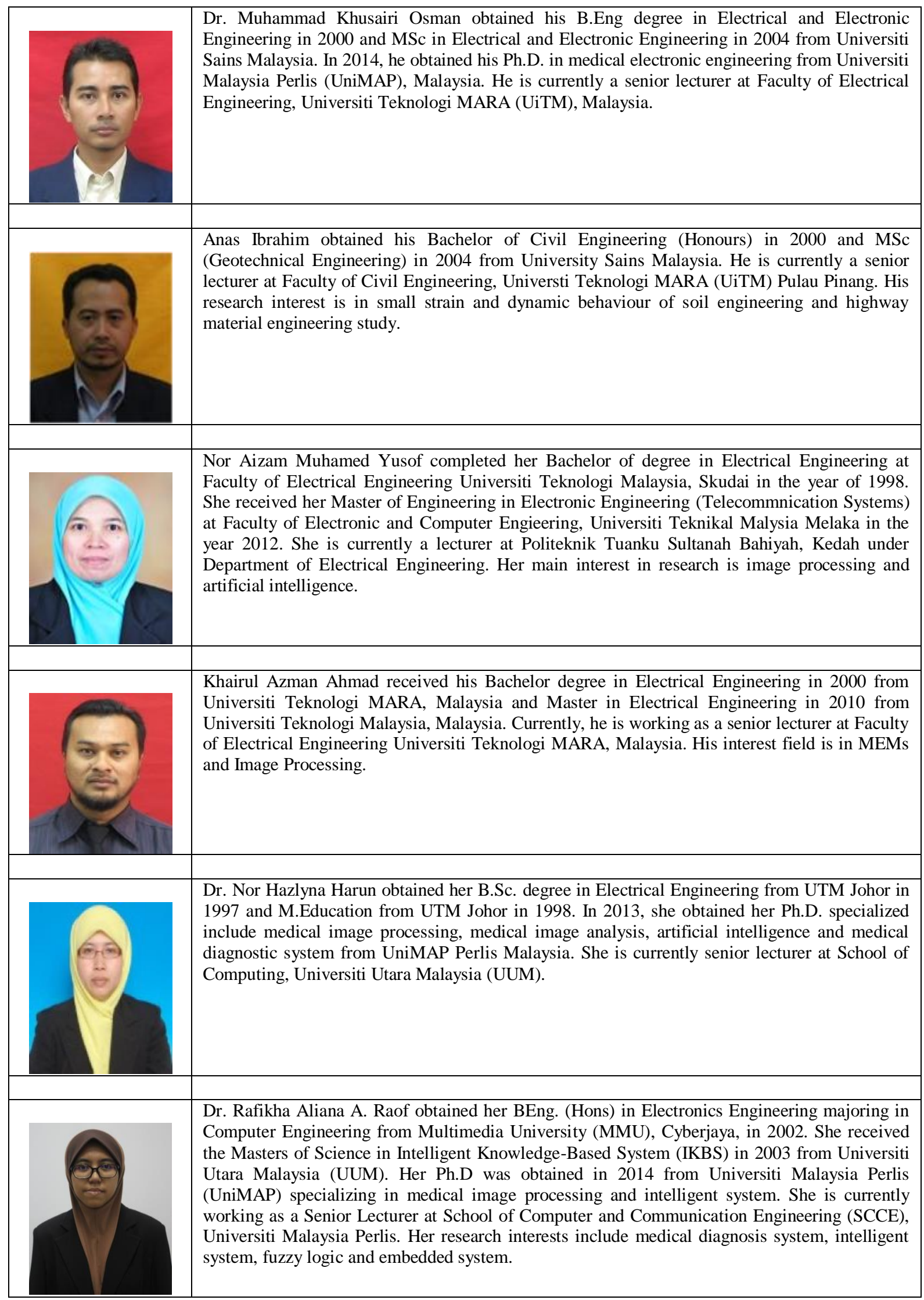

